\title{
ANÁLISE MULTICRITÉRIO PARA COMPOSIÇÃo DE PORTFÓLIO DE CURSOS DE TECNOLOGIA DA INFORMAÇÃO: UMA APLICAÇÃO DO MÉTODO ELECTRE-MOR
}

\author{
Igor Pinheiro de Araújo Costa (UFF) costa_igor@id.uff.br \\ José Victor de Pina Corriça \\ Daniel Augusto de Moura Pereira (UFCG) danielmoura@ufcg.edu.br \\ Carlos Francisco Simões Gomes (UFF) cfsg1@bol.com.br \\ Marcos dos Santos (IME) marcosdossantos_doutorado_uff@yahoo.com.br
}

\section{Resumo}

Este artigo tem por objetivo, mediante aplicação do método ELECTRE-MOr, apoiar o processo estratégico de formação de carteira de cursos de Treinamento e Desenvolvimento, ofertados por uma empresa do setor de treinamentos no Brasil, resultando em uma classificação de diversos cursos, visando apresentar aqueles que devem ser priorizados, mantidos ou descartados pela administração da empresa. Para a realização deste trabalho, foram consultados especialistas na área, que elencaram as alternativas de curso e os critérios a serem utilizados para a modelagem do problema no ELECREW-Mor. Os resultados encontrados mostraram que, dentre dezoito opções de cursos, seis deveriam ser priorizados, onze mantidos e um descartado, apresentando um algoritmo que pode ser aplicado em diversas situações estratégicas de empresas.

Palavras-Chaves: Análise Multicritério, ELECTRE-MOr, Tecnologia \& Desenvolvimento (T\&D).

\section{Introdução}

A crescente globalização dos negócios e a consequente exposição à concorrência mundial fizeram com que os conceitos de qualidade e competitividade tivessem uma nova importância e dimensão dentro das organizações (CHIAVENATO, 1996), e as fizeram reavaliar suas estratégias na área de Gestão de Pessoas, elevando o desempenho de seus colaboradores a fim de melhorar o relacionamento e satisfação dos clientes (CARVALHO, 2011).

Nesse contexto, o setor de Treinamento \& Desenvolvimento (T\&D) ganha mais destaque, considerando o terreno fértil que essa indústria vem apresentando. O mercado de treinamentos corporativos, especificamente, ganha uma importância ainda maior, e se mostra como uma 
oportunidade dentro do setor. De acordo com a última edição do relatório "Panorama do Treinamento no Brasil", a terceirização de ações de T\&D mantém um papel de importância fundamental na estratégia dentro das organizações. No ano de 2019, o valor investido em terceirização apresentou números muito expressivos: 50\% do orçamento das empresas (ABTD, 2019).

Sabendo-se que a indústria T\&D é uma realidade que já não oscila tão facilmente com a economia (ABTD, 2019), e dada a dimensão, perspectivas de futuro e gama de oportunidades dentro do mercado, é importante que organizações que atuam ou pretendem preencher lacunas dentro desse setor busquem se destacar de alguma forma. Nesse sentido, gerenciar seus stakeholders, sobretudo sua carteira de clientes e potenciais clientes, bem como analisar o relacionamento entre usuário e serviço, faz-se cada vez mais necessário e estratégico para o sucesso dessas organizações.

Neste sentido, este artigo tem como objetivo desenvolver, mediante aplicação do método de Apoio Multicritério à Decisão (AMD) ELECTRE-MOr, um algoritmo capaz de apoiar o processo estratégico de formação de carteira de cursos de T\&D oferecidos por uma empresa do setor de treinamentos no Brasil, resultando em uma divisão em classes de diversos cursos, indicando aqueles que devem ser priorizados, mantidos ou descartados pela administração da empresa.

Embora haja uma escassez geral de estudos que pesquisem os processos de tomada de decisão dos gerentes, na área de $T \& D$, essa lacuna é particularmente profunda na área de gestão de clientes (BAUER et al., 2013). Portanto, este trabalho contribui para a Administração Estratégica de empresas, principalmente em períodos de incerteza, como o atual momento, pois, a metodologia aqui apresentada pode ser aplicada em diversos problemas dos setores público e privado.

\section{Método ELECTRE-MOr}

O ELECTRE MOr é uma proposta de classificação de múltiplos critérios com entrada de pesos ordinal, que inclui múltiplos tomadores de decisão e distribui as alternativas em categorias predefinidas (COSTA et al., 2021).

Os procedimentos do ELECTRE MOr são desenvolvidos em duas etapas:

- Transformar preferências ordinais de critérios em um vetor de pesos de critérios; e 
- Integrar os critérios vetoriais de diferentes tomadores de decisão.

No procedimento de construção das relações de superação, o ELECTRE MOr utiliza uma adaptação do método Simple Aggregation of Preferences Expressed by Ordinal Vectors (SAPEVO) (GOMES et al., 2020) para gerar os pesos. Esta primeira etapa reúne dois passos:

Passo 1: a relação está associada à seguinte escala de cinco pontos, variando de -2 a 2 , conforme a Tabela 1:

Tabela 1 - Relação e escala

\begin{tabular}{|c|c|}
\hline Relação & Escala \\
\hline$\prec \prec$ é muito menos importante que & -2 \\
\hline$\prec$ é menos importante que & -1 \\
\hline$\approx$ é tão importante quanto & 0 \\
\hline$>$ é mais importante que & 1 \\
\hline$>>$ é muito mais importante que & 2 \\
\hline
\end{tabular}

Fonte: Os autores (2021)

Passo 2: essa relação associada a uma escala que permite transformar a matriz $\mathrm{Dk}=[\delta \mathrm{ij}]$, onde $\mathrm{k}=$ tomadores de decisão, em um vetor coluna [ $\left.\mathrm{v}_{\mathrm{i}}\right]$, de tal forma que (1):

$$
\sum_{j=1}^{m}\left(c_{i}\right), \text { para } i=1, \ldots, \text { me } k=1, \ldots, n
$$

Onde $c_{i}$ representa os graus atribuídos na avaliação paritária dos critérios, de acordo com a escala da Tabela 1. Terminado o processo de integração da matriz, é feita a normalização do vetor resultante, que garante a geração de valores não negativos (2):

$$
\overline{c_{l}}=\left(\frac{a_{i j}-\min a_{i j}}{\max a_{i j}-\min a_{i j}}\right)
$$

A partir do estudo dos métodos da família ELECTRE, foram desenvolvidos os índices de concordância, discordância e credibilidade. Na segunda etapa do método, esses índices são calculados de acordo com as seguintes fórmulas:

- Índice de concordância: A condição (p>q) deve ser atendida para que o resultado do cálculo não seja igual a zero (3); 
- Índice de concordância global (4);

$$
c_{j}\left(a, b_{h}\right)=\left\{\begin{array}{c}
0 \operatorname{seg}_{j}\left(b_{h}\right)-g_{j}(a) \geq p_{j}\left(b_{h}\right) \\
1 \operatorname{seg}_{j}\left(b_{h}\right)-g_{j}(a) \leq q_{j}\left(b_{h}\right) \\
\operatorname{se} p_{j} \geq g_{j}\left(b_{n}\right)-g_{j}(a)>q_{j} \\
1-\left(\frac{-g_{j}\left(b_{h}\right)+g_{j}(a)+q_{j}}{-p_{j}-q_{j}}\right)
\end{array}\right.
$$

$$
c\left(a, b_{h}\right)=\frac{\sum_{j \in F} w_{j} c_{j}\left(a, b_{h}\right)}{\sum_{j \in F} w_{j}}
$$

- Índice de discordância: A condição $(v>p)$ deve ser atendida para que o resultado do cálculo não seja igual a zero (5);

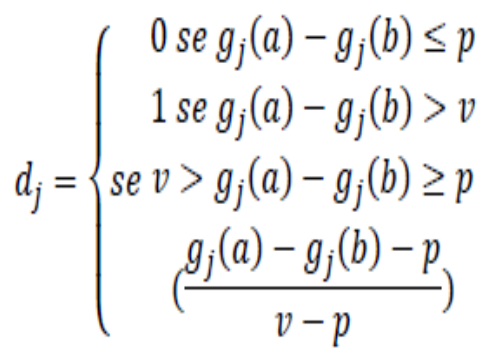

- Índice de credibilidade (6);

$$
\sigma\left(a, b_{h}\right)=c\left(a, b_{h}\right) \prod_{j \in F} \frac{1-d_{j}\left(a, b_{h}\right)}{1-c\left(a, b_{h}\right)}
$$

- Obtenção dos limites das classes: O método ELECTRE-MOr permite duas formas de estabelecer os perfis de classes - $b_{h}(7)$ e $b_{n}(8)$;

$$
b_{h}=g^{-}+h * k
$$

Onde $k=\frac{g^{*}-g^{-}}{h+1} ; g^{*}=\max _{j} g_{i j} ; g^{-}=\min _{j} g_{i j} ;$ h é o número de classes;

$$
b_{n}=g[(h+1-n) * L]
$$

Onde $L=\frac{j}{h+1}$; $n$ é o índice do perfil; j é o número de alternativas e h representa o número de limites das classes; e

- Nível de corte: A afirmação aSb $b_{h}$ significa que "a alternativa a não tem desempenho pior do que o perfil bh". Na validação da afirmação $\mathrm{aSb}_{\mathrm{h}}$, calcula-se um índice de credibilidade $\sigma\left(\mathrm{a}, \mathrm{b}_{\mathrm{h}}\right)$, o qual expressa o grau de confiança da declaração "a não é pior que $b_{h}$ ". Para definir a relação de sobreclassificação, é adotado o nível de corte $\lambda$ (9); 


$$
a S b_{h} \leftrightarrow \sigma\left(a, b_{h}\right) \geq \lambda
$$

A distribuição é realizada por meio de dois procedimentos:

- Otimista: consiste em comparar a alternativa sucessivamente à alternativa b, a partir do último perfil (categoria, classe); e

- Pessimista: consiste em comparar a alternativa a sucessivamente à alternativa b, partindo do primeiro perfil (categoria, classe), sendo esta a classificação mais exigente.

Devido ao fato de apresentar duas formas de obtenção dos limiares de classes predefinidas $\left(b_{h}\right.$ e $b_{n}$ ), o ELECTRE-MOr apresenta duas ordenações pessimistas e otimistas das alternativas. O fato de apresentar duas classificações após a aplicação do método permite uma análise de sensibilidade do resultado, conferindo maior transparência e robustez ao processo decisório (SOUZA; GOMES; DE BARROS, 2018).

\subsection{Aplicações do Métodos AMD em empresas}

O método ELECTRE-MOr vem sendo empregado para apoiar o processo de tomada de decisão em diversos problemas táticos, operacionais, logísticos e estratégicos, como na classificação de aviões-hospitais a serem empregados no combate à pandemia de COVID-19 (COSTA et al., 2020a); classificação de impressoras 3D para manufatura aditiva (DRUMOND et al.,., 2020); avaliação edafoclimática e econômica de regiões do Brasil para o plantio de mogno africano (MAÊDA et al., 2020); classificação de lanchas a serem adquiridas pela Marinha do Brasil no combate à COVID-19 no Amazonas (COSTA et al., 2020b); e em um estudo estratégico para a retomada econômica no município de Nilópolis, no estado do Rio de Janeiro (BERRIEL et al., 2020).

O cumprimento dos objetivos organizacionais está significativamente ligada ao efetivo controle dos portfólios de projetos (DANESH; RYAN; ABBASI, 2017). Existem muitos métodos de AMD disponíveis que podem ser usados para apoiar o processo decisório de gerenciamento de portfólio de projetos. Danesh et al., (2017) identificaram os pontos fortes e fracos dos métodos multicritério para auxiliar na tomada de decisões em portfólios. Dos mais de 100 métodos identificados em mais de 1.400 publicações, oito [Analytic Hierarchy Process (AHP), Analytic Network Process (ANP), Data Envelopment Analysis (DEA), Dominance-Based Rough Set Approach (DRSA), Elimination And Choice Expressing The Reality (ELECTRE), PreferenceRanking Organisation Method for Enrichment Evaluations (PROMETHEE), Technique for 
Order Preference by Similarity to an Ideal Solution (TOPSIS) e VlseKriterijumska Optimizacija I Kompromisno Resenje (VIKOR)] melhor se adequam a esse tipo de problema.

Kusi-Sarpong et al., (2016) aplicaram os métodos DEMATEL e AHP para examinar as práticas de gestão da cadeia de suprimentos ambientalmente sustentáveis na indústria de mineração de ouro de Gana.

O estudo realizado por Suifan et al., (2019) propôs um modelo integrado para analisar tradeoffs entre diferentes paradigmas de gestão da cadeia de suprimentos associados a prioridades competitivas. O estudo utilizou o método de entropia para derivar os pesos alternativos dos critérios de avaliação e o método TOPSIS para classificar alternativas viáveis por ordem de preferência e, em seguida, medir as trocas entre os objetivos conflitantes.

Vieira et al., (2020) estudaram as principais barreiras para implementar plenamente a logística reversa do lixo eletrônico no Brasil e priorizaram-nas sob uma abordagem multicritério, de acordo com as percepções das empresas, consumidores e do governo brasileiro. Foi aplicado o método Composition of Probabilistic Preferences (CPP) para analisar os resultados de cada grupo de tomadores de decisão. Guarnieri e De Almeida (2016), Galo et al., (2018) e Amoozad Mahdiraji et al., (2020) propuseram abordagens à categorização de alternativas com base no uso do ELECTRE TRI.

Após a revisão da literatura, verificou-se que os métodos de AMD vêm sendo muito empregados em gerenciamento de empresas. Este artigo apresenta um framework de distribuição de alternativas em classes predefinidas, apresentando como principal contribuição uma entrada de dados ordinal, tanto para obtenção dos pesos dos critérios, quanto para avaliação de critérios qualitativos. Ressalta-se que tais características não eram encontradas em métodos da família ELECTRE.

\section{Metodologia}

Para realização do presente estudo, foram consultados três especialistas da empresa objeto de estudo, com experiência na área de cursos de $\mathrm{T} \& \mathrm{D}$, que indicaram as alternativas de cursos consideradas como as mais importantes para a análise proposta, a saber: $\mathrm{A}_{1}$ - ITIL 4 Foundation, $\mathrm{A}_{2}$ - COBIT 2019 Foundation, $\mathrm{A}_{3}$ - Agile Scrum Foundation, A4 - Agile Scrum Master, A5 - Agile Scrum PO, $\mathrm{A}_{6}$ - Lean IT Foundation, $\mathrm{A}_{7}$ - DevOps Professional , $\mathrm{A}_{8}$ - DevOps Master, $\mathrm{A}_{9}$ Privacy \& Data Protection Foundation, $\mathrm{A}_{10}$ - Privacy \& Data Protection Essentials, $\mathrm{A}_{11}$ - 
Privacy \& Data Protection Practitioner , $\mathrm{A}_{12}$ - Information Security Foundation based on ISO 27001, A 13 - Information Security Management Professional based on ISO/IEC 27001, A14 - Catálogo de Serviços de TI, A 15 - Service Desk, , A16 - Orçamento de TI, A 17 - Roadmap de Governança de TI e $\mathrm{A}_{18}$ - Gestão de Fornecedores.

Ainda de acordo com os especialistas supracitados, os critérios a serem considerados na análise das alternativas devem ser os seguintes:

- $\mathrm{C}_{1}$ : Certificação vinculada: No mercado atual, as organizações dos mais diversos setores têm exigido, tanto de produto quanto de serviços, certificações como forma de qualificação e garantia de atendimento aos requisitos exigidos. Portanto, o fato de haver certificação aumenta a atratividade de um curso em detrimento a outro que não oferece certificado de conclusão;

- $\mathrm{C}_{2}$ : Pré-requisito: Condição prévia indispensável para se fazer um curso, por envolver conhecimentos prévios necessários. Verificou-Se que, quanto maior a exigência de prérequisitos, menor a demanda por determinado curso;

- $\mathrm{C}_{3}$ : Demanda no mercado: quantidade de um bem ou serviço que os consumidores desejam adquirir por um preço definido em um dado mercado, durante um dado período de tempo. Esse critério é muito importante, pois, para que a empresa aloque mais recursos e melhorias em prol de determinados cursos, a procura e retorno devem justificar tais esforços;

- $\mathrm{C}_{4}$ : Preço: Valor que deve ser dispendido pelo cliente para fazer determinado curso. Verificou-se que, quanto menor o preço, maior a procura;

- C5 $_{5}$ : Esforço interno: Empenho da empresa necessário para disponibilizar determinado curso, no que tange à preparação de instrutores, materiais didáticos e operacionalização das aulas.

As alternativas de cursos elencados foram avaliadas à luz dos cinco critérios estabelecidos e foram distribuídas em três classes, a saber:

- Classe A: Cursos que devem ser priorizados pela empresa, com incremento de investimentos e esforço interno;

- Classe B: Cursos que devem ser mantidos, sem alterações; e 
- Classe C: Cursos que podem ser excluídos da carteira de cursos oferecidos, sem prejuízos para a empresa.

\section{Resultados e Discussão}

\subsection{Aplicação do método ELECTRE-MOr}

O primeiro passo para a aplicação do método supracitado é a obtenção dos pesos dos critérios, mediante escala de relacionamento apresentada na Tabela 1 e equações (1) e (2). Em seguida, mediante adaptação do método SAPEVO, levando em conta as opiniões dos mesmos especialistas que definiram critérios e alternativas do problema, foram obtidos os pesos para os critérios, conforme mostra a Tabela 2.

Tabela 2 - Pesos dos critérios, obtidos após aplicação do ELECTRE-MOr

\begin{tabular}{|c|c|}
\hline Critério & Pesos (\%) \\
\hline C $_{1}$. Certificação vinculada & 18,13237 \\
\hline C $_{2}$. Pré-requisito & 0,271985 \\
\hline C $_{3}$. Demanda no mercado & 27,19855 \\
\hline C $_{4}$. Preço & 31,73164 \\
\hline C $_{5}$. Esforço interno & 22,66546 \\
\hline
\end{tabular}

Fonte: Os autores (2021)

Da Tabela 2, pode-se inferir que o critério com maior peso após análise dos especialistas foi o preço, com pouco mais de $30 \%$, seguido de demanda no mercado (27\%), esforço interno (22\%), certificação (18\%). O critério pré-requisito foi considerado como o menos importante pelos analistas. Os resultados encontrados após a modelagem mostraram-se coerentes com a visão de mercado, pois, é comum que os critérios de preço, demanda e esforço interno- representam a visão da empresa e dos clientes, no que tange à atratividade dos cursos.

\subsubsection{Avaliação das alternativas}

Para avaliação das alternativas, é obtida a matriz de performance, com as alternativas sendo avaliadas à luz de cada critério, conforme mostra a Tabela 3. 
Tabela 3 - Matrizes de performance

\begin{tabular}{|c|c|c|c|c|c|}
\hline CURSO & $\mathrm{C}_{1}$ & $\mathrm{C}_{2}$ & $\mathrm{C}_{3}$ & $\mathrm{C}_{4}$ & $\mathrm{C}_{5}$ \\
\hline $\mathrm{A}_{1}$ & 1 & 0 & 11,27451 & -480 & 9,803922 \\
\hline $\mathrm{A}_{2}$ & 1 & 0 & 8,088235 & -480 & 3,921569 \\
\hline $\mathrm{A}_{3}$ & 1 & 0 & 7,843137 & -460 & 8,088235 \\
\hline $\mathrm{A}_{4}$ & 1 & -1 & 6,127451 & -520 & 9,068627 \\
\hline $\mathrm{A}_{5}$ & 1 & -1 & 4,411765 & -520 & 2,696078 \\
\hline $\mathrm{A}_{6}$ & 1 & 0 & 0 & -420 & 0 \\
\hline $\mathrm{A}_{7}$ & 1 & 0 & 5,392157 & -520 & 2,696078 \\
\hline $\mathrm{A}_{8}$ & 1 & -1 & 5,392157 & -580 & 1,22549 \\
\hline $\mathrm{A}_{9}$ & 1 & 0 & 5,882353 & -520 & 2,45098 \\
\hline $\mathrm{A}_{10}$ & 1 & 0 & 5,882353 & -520 & 2,45098 \\
\hline $\mathrm{A}_{11}$ & 1 & -1 & 4,411765 & -580 & 1,715686 \\
\hline $\mathrm{A}_{12}$ & 1 & 0 & 6,127451 & -520 & 2,205882 \\
\hline $\mathrm{A}_{13}$ & 1 & -1 & 5,882353 & -520 & 2,205882 \\
\hline $\mathrm{A}_{14}$ & 0 & 0 & 5,637255 & -420 & 5,392157 \\
\hline $\mathrm{A}_{15}$ & 0 & 0 & 4,901961 & -420 & 5,392157 \\
\hline $\mathrm{A}_{16}$ & 0 & 0 & 4,411765 & -420 & 3,431373 \\
\hline $\mathrm{A}_{17}$ & 0 & 0 & 4,411765 & -420 & 4,166667 \\
\hline $\mathrm{A}_{18}$ & 0 & 0 & 3,921569 & -420 & 3,921569 \\
\hline $\mathbf{q}$ & 0,5 & 0,5 & 1 & 50 & 1 \\
\hline $\mathbf{p}$ & 1 & 1 & 3 & 100 & 3 \\
\hline $\mathbf{v}$ & 2 & 2 & 6 & 300 & 6 \\
\hline Pesos & 18,13237 & 0,271985 & 27,19855 & 31,73164 & 22,66546 \\
\hline $\mathbf{b}_{\mathbf{h} 2}$ & 0,67 & $-0,33$ & 7,52 & $-473,33$ & 6,54 \\
\hline $\mathbf{b}_{\mathbf{h 1}}$ & 0,33 & $-0,67$ & 3,76 & $-526,67$ & 3,27 \\
\hline $\mathbf{b}_{\mathbf{n} 2}$ & 1,00 & 0,00 & 5,88 & $-420,00$ & 4,17 \\
\hline $\mathbf{b}_{\mathbf{n 1}}$ & 1,00 & 0,00 & 4,90 & $-520,00$ & 2,45 \\
\hline & & & & & \\
\hline
\end{tabular}

Fonte: Os autores (2021)

Os critérios demanda no mercado $\left(\mathrm{C}_{3}\right)$ e esforço interno $\left(\mathrm{C}_{5}\right)$ também foram avaliados pelos especialistas mediante aplicação da adaptação do método SAPEVO, por se tratarem de critérios qualitativos, devido ao fato de ser uma tarefa árdua definir, dentre 18 alternativas, uma escala cardinal de preferências. Os critérios certificação $\left(\mathrm{C}_{1}\right)$ e pré-requisito $\left(\mathrm{C}_{2}\right)$ foram avaliados por uma escala binária, na qual 1 representa a ocorrência e 0 significa a não-ocorrência em cada critério. Os critérios pré-requisito $\left(\mathrm{C}_{2}\right)$ e preço $\left(\mathrm{C}_{4}\right)$ encontram-se com valores negativos na matriz, por serem considerados monotônicos de custo, ou seja, busca-se minimizar seus valores. Os limiares de Preferência Fraca (q), Preferência estrita (p) e Veto (v) foram definidos mediante consenso entre os especialistas. Já os valores de $b_{h}$ e $b_{n}$ foram obtidos pela aplicação das equações (7) e (8). 


\subsubsection{Resultados obtidos}

Após aplicação de todos os passos do ELECTRE-MOr, foram obtidas as classificações otimistas e pessimistas para as duas formas de normalização $\left(b_{h}\right.$ e $\left.b_{n}\right)$, para um nível de corte $\lambda$ igual a 0,65 . Por definição, o valor de $\lambda$ varia de 0,5 a 1 e, quanto maior seu valor, mais exigente é a distribuição das alternativas nas classes predefinidas (COSTA et al., 2021). Neste artigo, foi aplicado o valor de 0,65 para o valor de corte, por apresentar uma boa discriminação na classificação das alternativas mediante aplicação da Eq. 9. Assim, obtém-se a distribuição das alternativas nas três classes predefinidas (Tabela 4).

Tabela 4 - Resultados obtidos após aplicação do método

\begin{tabular}{|c|c|c|c|c|c|}
\hline \multicolumn{2}{|c|}{$\mathrm{b}_{\mathrm{h}}$} & \multicolumn{3}{|c|}{$\mathrm{b}_{\mathrm{n}}$} \\
\hline Alternativa & Pessimista & Otimista & Alternativa & Pessimista & Otimista \\
\hline $\mathrm{A}_{1}$ & $\mathrm{~A}$ & $\mathrm{~A}$ & $\mathrm{~A}_{1}$ & $\mathrm{~A}$ & $\mathrm{~A}$ \\
\hline $\mathrm{A}_{2}$ & $\mathrm{~A}$ & $\mathrm{~A}$ & $\mathrm{~A}_{2}$ & $\mathrm{~A}$ & $\mathrm{~A}$ \\
\hline $\mathrm{A}_{3}$ & $\mathrm{~A}$ & $\mathrm{~A}$ & $\mathrm{~A}_{3}$ & $\mathrm{~A}$ & $\mathrm{~A}$ \\
\hline $\mathrm{A}_{4}$ & $\mathrm{~A}$ & $\mathrm{~A}$ & $\mathrm{~A}_{4}$ & $\mathrm{~A}$ & $\mathrm{~A}$ \\
\hline $\mathrm{A}_{5}$ & $\mathrm{~B}$ & $\mathrm{~B}$ & $\mathrm{~A}_{5}$ & $\mathrm{~B}$ & $\mathrm{~B}$ \\
\hline $\mathrm{A}_{6}$ & $\mathrm{C}$ & $\mathrm{B}$ & $\mathrm{A}_{6}$ & $\mathrm{C}$ & $\mathrm{C}$ \\
\hline $\mathrm{A}_{7}$ & $\mathrm{~B}$ & $\mathrm{~B}$ & $\mathrm{~A}_{7}$ & $\mathrm{~B}$ & $\mathrm{~B}$ \\
\hline $\mathrm{A}_{8}$ & $\mathrm{~B}$ & $\mathrm{~B}$ & $\mathrm{~A}_{8}$ & $\mathrm{~B}$ & $\mathrm{~B}$ \\
\hline $\mathrm{A}_{9}$ & $\mathrm{~A}$ & $\mathrm{~A}$ & $\mathrm{~A}_{9}$ & $\mathrm{~B}$ & $\mathrm{~B}$ \\
\hline $\mathrm{A}_{10}$ & $\mathrm{~A}$ & $\mathrm{~A}$ & $\mathrm{~A}_{10}$ & $\mathrm{~B}$ & $\mathrm{~B}$ \\
\hline $\mathrm{A}_{11}$ & $\mathrm{~B}$ & $\mathrm{~B}$ & $\mathrm{~A}_{11}$ & $\mathrm{~B}$ & $\mathrm{~B}$ \\
\hline $\mathrm{A}_{12}$ & $\mathrm{~A}$ & $\mathrm{~A}$ & $\mathrm{~A}_{12}$ & $\mathrm{~B}$ & $\mathrm{~B}$ \\
\hline $\mathrm{A}_{13}$ & $\mathrm{~A}$ & $\mathrm{~A}$ & $\mathrm{~A}_{13}$ & $\mathrm{~B}$ & $\mathrm{~B}$ \\
\hline $\mathrm{A}_{14}$ & $\mathrm{~A}$ & $\mathrm{~A}$ & $\mathrm{~A}_{14}$ & $\mathrm{~A}$ & $\mathrm{~A}$ \\
\hline $\mathrm{A}_{15}$ & $\mathrm{~A}$ & $\mathrm{~A}$ & $\mathrm{~A}_{15}$ & $\mathrm{~A}$ & $\mathrm{~A}$ \\
\hline $\mathrm{A}_{16}$ & $\mathrm{~B}$ & $\mathrm{~B}$ & $\mathrm{~A}_{16}$ & $\mathrm{~A}$ & $\mathrm{~A}$ \\
\hline $\mathrm{A}_{17}$ & $\mathrm{~B}$ & $\mathrm{~B}$ & $\mathrm{~A}_{17}$ & $\mathrm{~A}$ & $\mathrm{~A}$ \\
\hline $\mathrm{A}_{18}$ & $\mathrm{~B}$ & $\mathrm{~B}$ & $\mathrm{~A}_{18}$ & $\mathrm{~A}$ & $\mathrm{~A}$ \\
\hline
\end{tabular}

Fonte: Os autores (2021)

Analisando os resultados, observa-se quatro classificações, o que permite uma análise mais transparente e robusta. Para a clusterização das alternativas, visando obter a divisão dos cursos em três classes definidas, foi utilizado o seguinte critério: Uma alternativa que apresenta resultado compatível com uma determinada classe em três ou quatro cenários, é alocada nesta classe; uma alternativa que apresenta determinada classificação em dois dos quatro cenários será alocada na classe mais baixa. 
Para ilustrar a clusterização, a alternativa $\mathrm{A}_{1}$ será alocada na classe $\mathrm{A}$, pois apresenta tal desempenho nos quatro cenários. A6 será alocada na classe $\mathrm{C}$, uma vez que apresenta três das quatro classificações compatíveis com esta classe. Já a alternativa $\mathrm{A}_{16}$ ocupará a classe B, pois apresenta duas ordenações na classe A e duas na B, sendo esta a pior classificação apresentada pela alternativa. Portanto, a classificação das alternativas de cursos consta na Tabela 5.

Tabela 5 Distribuição dos cursos nas três classes predefinidas

\begin{tabular}{|c|c|}
\hline Cursos & Classe \\
\hline $\begin{array}{l}\mathrm{A}_{1} \text { - ITIL } 4 \text { Foundation, } \mathrm{A}_{2} \text { - COBIT } 2019 \text { Foundation, } \mathrm{A}_{3} \text { - Agile Scrum Foundation, } \mathrm{A}_{4} \text { - } \\
\text { Agile Scrum Master, } \mathrm{A}_{14} \text { - Catálogo de Serviços de TI, } \mathrm{A}_{15} \text { - Service Desk }\end{array}$ & A \\
\hline $\begin{array}{l}\mathrm{A}_{5} \text { - Agile Scrum PO, } \mathrm{A}_{7} \text { - DevOps Professional, } \mathrm{A}_{8} \text { - DevOps Master, } \mathrm{A}_{9} \text { - Privacy \& Data } \\
\text { Protection Foundation, } \mathrm{A}_{10} \text { - Privacy \& Data Protection Essentials, } \mathrm{A}_{11} \text { - Privacy \& Data } \\
\text { Protection Practitioner, } \mathrm{A}_{12} \text { - Information Security Foundation based on ISO } 27001, \mathrm{~A}_{13} \text { - } \\
\text { Information Security Management Professional based on ISO/IEC } 27001, \mathrm{~A}_{16} \text { - Orçamento } \\
\text { de TI, } \mathrm{A}_{17} \text { - Roadmap de Governança de TI e } \mathrm{A}_{18} \text { - Gestão de Fornecedores }\end{array}$ & B \\
\hline $\mathrm{A}_{6}$ - Lean IT Foundation & $\mathrm{C}$ \\
\hline
\end{tabular}

Fonte: Os autores (2021)

Analisando os resultados obtidos, observa-se que os cursos $\mathrm{A}_{1}, \mathrm{~A}_{2}, \mathrm{~A}_{3}, \mathrm{~A}_{4}, \mathrm{~A}_{14}$ e $\mathrm{A}_{15}$ obtiveram classificação A, podendo ser considerados como os cursos que devem ser priorizados pela empresa, por apresentarem, após análise dos especialistas e aplicação da metodologia proposta, os melhores desempenhos entre as alternativas. Ressalta-se que, das dezoito alternativas avaliadas, apenas seis obtiveram desempenho que justificasse incremento de investimento e esforço em prol de cada uma.

$A$ alternativa $A_{6}$ foi a única que apresentou desempenho de classe $C$, o que justificaria sua exclusão da carteira de cursos oferecidos pela empresa. As 11 alternativas restantes apresentaram desempenho condizente com a classe B, o que justificaria a manutenção dessas opções dentre os cursos oferecidos.

Abordando os motivos que levaram as 6 alternativas a ocuparem a classe mais alta, observa-se que tais cursos destacam-se nos critérios com os maiores pesos obtidos após análise dos especialistas - Menor preço, maior demanda de mercado e menor esforço interno por parte da empresa -, o que mostra que os resultados alcançados apresentam bastante coerência.

Já as alternativas que alcançaram o desempenho relacionado à classe B apresentaram, de um modo geral, desempenho intermediário nos critérios analisados. Em alguns casos, apesar de se 
destacarem em determinado critério, tal desempenho foi contrabalanceado por um baixo grau em outro, o que justifica a alocação das 11 alternativas em uma classe intermediária.

Por fim, tratando os motivos que lavaram o curso $\mathrm{A}_{6}$ na pior classe possível, nota-se que esta apresentou o pior desempenho absoluto nos dois critérios com o maior peso na análise, o que justificaria sua exclusão do quadro de cursos oferecidos pela empresa.

\section{Considerações Finais}

A aplicação do método ELECTRE-MOr considerou a avaliação de três especialistas diferentes, com base nos critérios em questão e dos pesos provenientes de suas avaliações, tendo interferido sobre o resultado final. Tendo em vista o objetivo do artigo - apoiar o processo decisório no que tange à priorização de cursos de T\&D-, o grande peso atribuído aos critérios preço e demanda se justifica, mostrando que a utilização do método auxiliou a obtenção de um valor cardinal de preferência entre os critérios mediante uma entrada de dados ordinal.

Ressalta-se que o método aqui utilizado se mostrou eficiente para a análise proposta, possibilitando entrada de pesos ordinais, levando em consideração a opinião de múltiplos decisores, tanto na obtenção dos pesos, quanto na elicitação dos limiares de preferência, veto e limites das classes predefinidas. Além disso, a apresentação de duas ordenações pessimistas e otimistas, permite verificar o comportamento das alternativas em diversos cenários, com a possibilidade de escolha de diversos parâmetros de análise, seja pela consideração do maior número de ocorrências de classificações, como foi feito neste trabalho, ou pela consideração de apenas uma das duas formas de ordenação. Essa característica fornece uma informação adicional ao tomador de decisão.

Em face ao exposto, pode-se afirmar que o objetivo do artigo foi atingido, com a apresentação de uma distribuição para priorização de cursos de T\&D ofertados pela empresa. Ressalta-se que a metodologia apresentada nesta pesquisa pode ser aplicada em diversas outras situações do meio corporativo, tornando o método extremamente útil para resolução de problemas reais de níveis tático, operacional ou estratégico, sendo assim uma de grande utilidade voltada para o apoio à tomada de decisão de alto nível.

\section{Referências}

ABTD. Panorama do treinamento no brasil: indicadores e tendências em gestão doT\&D. Associação Brasileira 
de Treinamento e Desenvolvimento, 2019.

AMOOZAD MAHDIRAJI, H.; HAFEEZ, K.; RAZAVI HAJIAGHA, S. H. Business process transformation in financial market: A hybrid BPM-ELECTRE TRI for redesigning a securities company in the Iranian stock market. Knowledge and Process Management, v. 27, n. 3, p. 211-224, 2020.

BAUER, J. C. et al. Managerial decision making in customer management: adaptive, fast and frugal? Journal of the Academy of Marketing Science, v. 41, n. 4, p. 436-455, 2013.

BERRIEL, P. C. et al. Retomada econômica no município de Nilópolis durante a pandemia de COVID-19: uma análise a partir do método ELECTRE-MOr. Congreso Internacional XXXI EPIO - XXXIII ENDIO y REDM IX, 2020.

CARVALHO, J. C. B. DE. A necessidade de investimento em T\&D no comércio varejista da cidade de Rio Branco para atender a satisfação do cliente. 2011.

CHIAVENATO, I. Como transformar RH de um centro de despesas em um centro de lucro. [s.l.] Makron Books, 1996.

COSTA, I. P. DE A. et al. Desenvolvimento axiomático e implementação computacional do método híbrido ELECTRE-MOr: escolha de avião-hospital no apoio ao combate à COVID-19. 15 $^{\circ}$ Congresso Brasileiro de Sistemas, 2020a.

COSTA, I. P. DE A. et al. Desenvolvimento axiomático e implementação computacional do método híbrido ELECTRE-MOr: apoiando à decisão da Marinha do Brasil na aquisição de lanchas no combate à COVID-19. Congreso Internacional XXXI EPIO - XXXIII ENDIO y RED-M IX 2020, 2020 b.

COSTA, I. P. DE A. et al. Choosing flying hospitals in the fight against the COVID-19 pandemic: structuring and modeling a complex problem using the VFT and ELECTRE-MOr methods. IEEE Latin America Transactions, v. 19, n. 6, p. 1099-1106, 2021.

DANESH, D.; RYAN, M. J.; ABBASI, A. A systematic comparison of multi-criteria decision making methods for the improvement of project portfolio management in complex organisations. International Journal of Management and Decision Making, v. 16, n. 3, p. 280-320, 2017.

DRUMOND, P. et al. Aplicação do método ELECTRE-MOr na manufatura aditiva: classificação de impressora 3S do tipo Fused Deposition Modeling (FDM). Congreso Internacional XXXI EPIO - XXXIII ENDIO y RED-M IX, 2020.

GALO, N. R.; CALACHE, L. D. D. R.; CARPINETTI, L. C. R. A group decision approach for supplier categorization based on hesitant fuzzy and ELECTRE TRI. International Journal of Production Economics, v. 202, p. 182-196, 2018.

GOMES, C. F. S. et al. SAPEVO-M: a group multicriteria ordinal ranking method. Pesquisa Operacional, v. 40, 2020.

GUARNIERI, P.; DE ALMEIDA, A. T. A Multicriteria Decision Model for Collaborative Partnerships in Supplier Strategic Management. Journal of Advanced Manufacturing Systems, v. 15, n. 3, p. 101-131, 2016.

KUSI-SARPONG, S.; SARKIS, J.; WANG, X. Green supply chain practices and performance in Ghana's mining industry: A comparative evaluation based on DEMATEL and AHP. International Journal of Business Performance and Supply Chain Modelling, v. 8, n. 4, p. 320-347, 2016.

MAÊDA, S. M. DO N. et al. Avaliação edafoclimática e econômica de regiões para o plantio de mogno africano: uma abordagem à luz do método ELECTRE-MOr. Congreso Internacional XXXI EPIO - XXXIII ENDIO y RED-M IX, 2020.

SOUZA, L. P. DE; GOMES, C. F. S.; DE BARROS, A. P. Implementation of new hybrid AHP-TOPSIS-2N method in sorting and prioritizing of an it CAPEX project portfolio. International Journal of Information 
Technology \& Decision Making, v. 17, n. 04, p. 977-1005, 2018.

SUIFAN, T.; ALAZAB, M.; ALHYARI, S. Trade-off among lean, agile, resilient and green paradigms: An empirical study on pharmaceutical industry in Jordan using a TOPSIS-entropy method. International Journal of Advanced Operations Management, v. 11, n. 1-2, p. 69-101, 2019.

VIEIRA, B. O. et al. Prioritizing barriers to be solved to the implementation of reverse logistics of e-waste in Brazil under a multicriteria decision aid approach. Sustainability (Switzerland), v. 12, n. 10, 2020. 